\author{
Sensitivity of an Energy Doubler \\ Dipole to Beam Induced Quenches
}

B.Cox, P. O. Mazur and A. Van Ginneken

November 1978

\begin{abstract}
In a series of tests in the $\mathrm{P}$-West proton beam a doubler dipole (E22-12) was exposed to various intensity $400 \mathrm{GeV} / \mathrm{c}$ beams with several types of spills. Results are presented for the sensitivity of this magnet to beam induced quenches and operation of the superconducting dipole in a proton beam over a three month period is di scussed.
\end{abstract}


The question of the amount of beam loss which will quench a superconducting magnet has taken on special importance at this time because of the plans for the $1000 \mathrm{GeV}$ Energy Doubler. A question of secondary importance but of interest is what operational difficulties may be encountered during the long term operation of superconducing magnets in a beam line. For a period of three months an Energy Doubler dipole (E22-12) was operated in the P-West beam line with proton beams in the range $10^{7}-10^{12}$ passing through it. During this time a special series of tests was conducted to determine the amounts of $\mu \mathrm{sec}, 1 \mathrm{msec}$ and .5 second slow spill beam loss that would induce quenches in this magnet.

The geography of the installation is shown in figures la and Ib and the schematic of the helium refrigeration system used to cool this magnet is shown in Fig. 2a. As shown, the installation was situated in the P-West line and the doubler dipole in normal operation performed the function of bending the incident $400 \mathrm{GeV}$ proton beam $10.375 \mathrm{mradians}$ onto the High Intensity Laboratory target. The nominal current required for this standard operation was approximately 2300 amps which was provided by a $500 \mathrm{kw}$ Transrex power supply. The helium refrigeration for this magnet was provided by a CTI 1400 refrigerator which in standard Iiquefaction mode operation produced 30-40 liters/hour into a 450 liter dewar pressurized to 4.5 psig. The dewar pressure was then raised to 8 psig and liquid was transported from this dewar via a 100 foot transfer line to a counterflow heat exchanger (subcooler) before entering the doubler dipole coil region as a single phase fluid. In 
standard operation the pressure of this region was 23 psia corresponding to a saturation temperature of $4.73^{\circ} \mathrm{K}$. The helium was subcooled by $\sim 0.05^{\circ} \mathrm{K}$. The single phase liquid then passed through the magnet to a Joule-Thompson valve into the two phase region, which is in thermal contact with the single phase region. The helium, at this point a boiling two phase mixture, passed back through the subcooler and to the refrigerator cold return. A battery of three compressors (Schramm, CTI, and APCI) provided 10.8 $\mathrm{g} / \mathrm{sec}$ at $240 \mathrm{psig}$ for the CTI 1400 during an average operating period. The Joule-Thompson valve was operated in both manual and automatic modes. In automatic mode, the valve was controlled using the temperature difference between the gas returning from the subcooler to the refrigerator and its saturation temperature. This was accomplished by measuring the pressure difference between the returning gas and a vapor pressure bulb in the gas stream. A pressure difference of $1-2$ psi, corresponding to a temperature of $0.05-0.1^{\circ} \mathrm{K}$ above saturation, was used. It was found that, due to the long response times of the system to changes in flow lof order 5 minutes), that more stable operation could be obtained by setting the J-T valve manually. A 1-2 psi pressure drop across the valve resulted in stable operation with a constant dewar pressure of about 8 psig and a refrigerator return pressure of about 6 psig. Heat transfer in this early version of the Doubler magnet was insufficient to maintain subcooling, and the vapor pressure thermometer just before the J-T valve indicated the helium there was saturated. Recovery from the various types of quenches (either 
accidental or intentional) with this system seemed to depend mainly on the current at which the magnet was operating. The time of recovery varied roughly from 5 minutes at 2500 amps to 15 minutes at 3500 amps. The quench detection and energy dump circuitry is shown schematically in Fig. $2 b$ along with a typical current decay curve for a typical beam induced quench. E22-12 was an early model of an Energy Doubler magnet which had been trained to 4800 amps and normally would have operated with a $20^{\circ} \mathrm{K}$ helium shield. Because of a thermal short in the lead box from this shield to the $4^{\circ} \mathrm{K}$ two phase region it was found that the most efficient mode in which to operate the magnet was with the shield shut off. In this mode one shift operation was possible before the system would have to be shut down to refill the dewar either with the refrigerator or from reserve dewars. During the three month period we performed over 35 cool downs from $30^{\circ} \mathrm{K}$ to $4^{\circ} \mathrm{K}$ and approximately 10 cool downs from $300^{\circ} \mathrm{K}$ to $4^{\circ} \mathrm{K}$. The magnet was subjected to approximately 100 quenches, 60 of which were beam induced either in normal 2300 amp operation or at the other magnet currents used during the special quench tests discussed below. It is noteworthy that only two or three of these quenches during the three months of operation could be attributed to accelerator malfunctions such as beam missteering or accidental high intensity pulses. The position stability of the beam was remarkable and the intensity stability (less than a factor of \pm 3 ) of the beam was reasonable. When the magnet was set to target protons and was left unattended no drifting occurred and, as mentioned, only a few accidental quenches were experienced. No 
discernable deterioration of the doubler magnet occurred durinc this usage. This experience leads us to be optimistic about routine operations of Doubler magnets in external beam line: (exclusive of the possibility of beam induced quenches).

To explicitly measure the quench properties of this doubler dipole, time was set aside for a set of tests of this magnet when exposed to slow, msecond, and usecond spills of varying intensities. In these tests we attempted the following measurements:

1) Protons required to induce a quench using slow, msecond, and $\mu$ second spills with the $400 \mathrm{GeV}$ beam impacting the coil region in a grazing trajectory (Geometry I, Fig. 3a).

2) Protons required to induce a quench with the $400 \mathrm{GeV}$ beam impacting the upstream end of the magnet in an island region (Geometry II, Fig. 3b).

3) The protons required in Geometry II to induce a quench with the $400 \mathrm{GeV}$ beam as a function of magnet current.

4) Protons required in Geometry II to quench the magnet for different beam spot sizes.

Figure $3 \mathrm{c}$ shows the relative position of the coils and the bore tube. The impact angle of the beam on the coil region in the grazing configuration of Geometry $I$ is 10.75 mradians and the impact region starts approximately 60 inches from the downstream end of the magnet when the magnet is run at 3500 amps. A crude measure of the relative energy dump at various intensities was 
provided by a pair of coupled ion chambers (loss monitors) positioned on the downstream end of the doubler magnet. The temperature of the magnet was monitored by a vapor pressure thermorneter situated in the single phase helium volume at the downstream end of the magnet. Incident beam intensity was measured by a battery of ion chambers and secondary emmission monitors upstream of the dipole.

Figure 4 shows the response in Geometry $I$ of the loss monitor vs. the $400 \mathrm{GeV}$ proton beam intensity. As shown in Fig. 4, the quench points of E22-12 were measured a number of times for four different types of spill; $.5 \mathrm{sec}$ slow spill, $1 \mathrm{msec}$ fast spill, $\mu \mathrm{sec}$ spill with four 1.6 usec booster bunches spread uniformly around the main $r$ ing and usec spill with two $1.6 \mu$ sec booster bunches adjacent to one another. A Monte Carlo shower calculation was performed to estimate the energy densities of the hadronic and electromagnetic showers caused by the impact of the beam in both Geometry I and II. An elliptical beam spot of $18 \times 15 \mathrm{~mm}$ with a gaussian distribution along the principal axis was used in the calculation. Approximately $95 \%$ of the beam was contained within this ellipse. This profile was consistent with the beam profiles measured on the beam monitors during all phases of the tests. For Geometry I the largest energy density is predicted to occur in the inner turn at the midplane and is approximately $.75 \mathrm{GeV} / \mathrm{cm}^{3}$ per incident proton. It is estimated that the energy density is accurate to approximately $25 \%$. The temperature of the single phase coil region was monitored to be $4.78 \pm .05^{\circ} \mathrm{K}$ before beam impact 
for all the quenches. The magnetic field in the region of maximum energy density at 3500 amps is estimated to be $3.5 \mathrm{~T}$. The variation of short sample limit with temperature for the doubler conductor is such that at $6.2^{\circ} \mathrm{K}$ the critical current will be exceeded for this magnet at 3500 amps. This corresponds to an allowable $\Delta \mathrm{T}$ of $1.4^{\circ} \mathrm{K}$ which in turn corresponds to an allowable energy density of 9.8 mjoules $/ \mathrm{cm}^{3}$. From the results of the shower calculations the maximum beam that could impact the coil without quenching (in the absense of heat transfer mechanisms) would be approximately $8 \times 10^{7}$ protons. As shown in Fig. 4, slow spill quenches occur at approximately $4-5$ times this level at $3 \times 10^{8}$ protons per .5 seconds. When the msecond fast spill was measured we saw a marked increase in the sensitivity of the magnet with the quenches occurring at $10^{8}$ protons. Finally for the usecond spills we saw a slight increase in sensitivity with the quenches occuring typically at 7-8 $\times 10^{7}$ protons. As shown in Fig. 4 there was a further small increase in sensitivity when the usecond spill structure was changed from four 1.6 usecond bunches spread evenly over 20

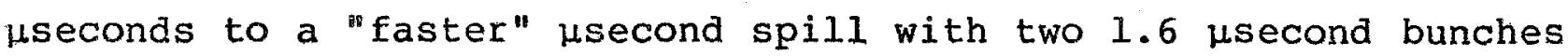
adjacent to one another. While this change is probably not significant there still may be some heat transfer effects present at this level. The dotted line shows the upper limit on acceptable targeted beam in this geometry which is predicted by our simple calculation. As can be seen this is very close to the quench points measured with the usecond and msecond spills. 
A second measurement of the sensitivy of E22-12 was done in the beam impact geometry shown as Geometry II in Fig. 3b with the .5 second slow spill. The results of this "perpendicular" impact measurement are shown in Fig. 5. A different slope of loss monitor vs. beam intensity is obtained, of course, since the targeting geometry is different. The major observation is that the quench point occurs at a factor of 10 higher intensity $\left(\sim 2-3 \times 10^{9}\right.$ protons). This is because the beam is initially striking an island and not a coil region in the dipole. This interpretation is approximately supported by the result of a shower calculation for Geometry II which predicts a maximum energy density roughly a factor of 10 less than the maximum density calculated for Geometry I. This shower calculation is analogous to that performed for Geometry I. The maximum energy density in the coil region is calculated to be $30-40 \mathrm{~cm}$ into the magnet and was $.078 \mathrm{GeV} / \mathrm{cm}^{3}$ per incident proton. This energy density was roughly independent of the magnetic field for $3.5 \mathrm{~T}$ and $0.4 \mathrm{~T}$. In Geometry II we tried two other measurements. In order to determine whether the magnet was at a uniform temperature and to try to detect the possible existence of gas pockets in the coil region the beam was targeted both above and below the gap. As Fig. 5 shows, there is a slight increase in sensitivity when the beam is targeted in the upper position. This is probably within the scatter of the quench points for identical geometries. The second type of measurement was made by increasing the spot size to lower the energy density of the shower. A slight decrease in sensitivity is seen for this larger spot. Once again the decrease is slight but seems to go in the proper direction. 
As a final measurement of the sensitivity of this dipole to beam, the variation of the slow spill quench point with magnet current was measured. The results are shown in Fig. 6 . Between 420 amps and 3500 amps there is an increase of an order of magnitude in the sensitivity of E22-12. In exercising this magnet without beam we found that spontaneous or training type quenches began to occur between 3900 and 4000 amps (the magnet had been trained to 4800 amps but at a lower temperature). When we set the magnet at 4000 amps we found a marked increase in sensitivity with the magnet quenching at the $10^{7}$ proton level. This phenomenon was reproducible.

In conclusion, we have measured the quench behavior of an Energy Doubler dipole. The results of our measurements appear (at the level thus far studied) to indicate that the quench behavior of this magnet can be predicted by a shower calculation and the assumption is approximatly correct that no heat transfer mechanism is rapid enough to carry off an appreciable amount of beam energy in the case of the usecond spill. In the case of .5 second slow spill approximately an order of magnitude more beam can be tolerated than in the case of $\mu$ second spill. There is also a systematic variation of the quench point of the doubler dipole with field which allows an order of magnitude more slow spill beam to be scraped at 420 amps (Energy Doubler injection current) than at 3500 amps. These measurements are summarized in Table $I$.

Finally, a number can be quoted for the sensitivity of the doubler magnet to beam. The instantaneous energy density must be kept below 
$9.8 \mathrm{mj} / \mathrm{cm}^{3}$ or $950 \mu \mathrm{j} / \mathrm{linear} \mathrm{cm}$ of 23 strand for 3500 at $4.8^{\circ} \mathrm{K}$ doubler conductor

$5.2 \mathrm{mj} / \mathrm{cm}^{3}$ or $500 \mu j / 1$ inear $\mathrm{cm}$ of 23 strand for 4250 at $4.3^{\circ} \mathrm{K}$ doubler conductor operating parameters)

We would like to acknowledge many conversations with Helen Edwards which helped tremendously in defining and clarifying this problem. 
TABLE I

Type of Spill

Magnet Current (amps)

3500

3500

3500

$\mu$ sec, 4 bunches

- Geometry I

usec, 2 bunches

- Geometry I

.5 sec-Geometry II
3500

420

1000

1500

2000

2500

3000

3500

4000
Beam Intensity

$3 \times 10^{8}$

I. $x \cdot 10^{8}$

$.7 \times 10^{8}$

$.5 \times 10^{8}$

$4 \times 10^{10}$

$1.7 \times 10^{10}$

$1.2 \times 10^{10}$

$7 \times 10^{9}$

$4.5 \times 10^{9}$

$3.7 \times 10^{9}$

$2 \times 10^{9}$

$2 \times 10^{7}$ 

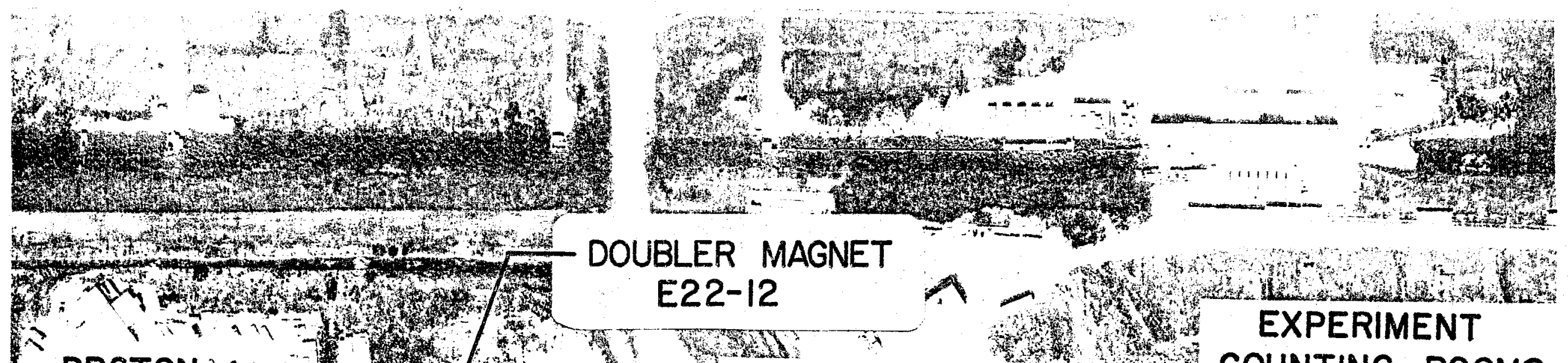

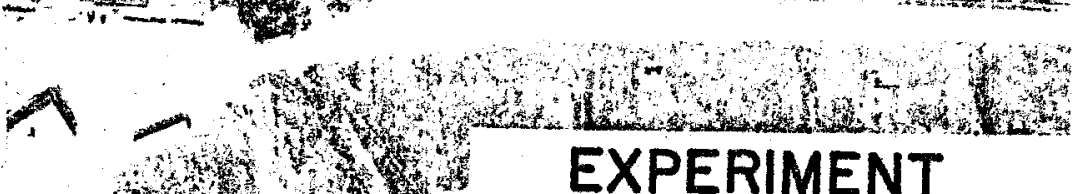

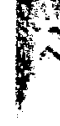

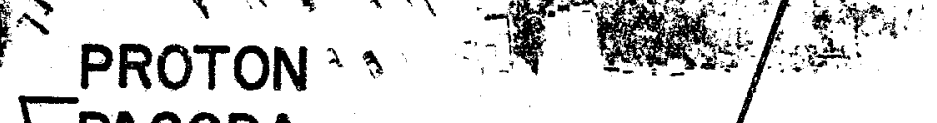

IPAGODA.

Hen

Whit

(1)

3.

N.20

(स)

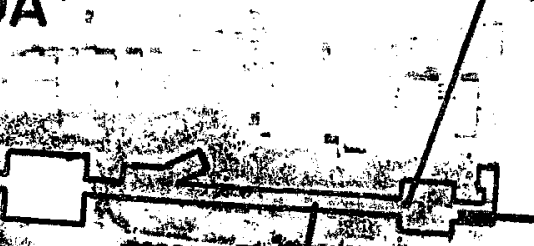

स W WX How mbs

EXPERIMENT

\section{SECONDARY BEAM}

COUNTING ROOMS

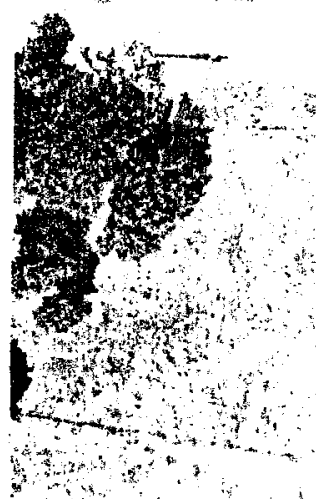

PROTON

TARGETING

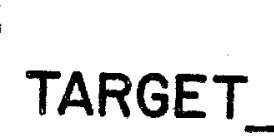

:SHIELDING - -
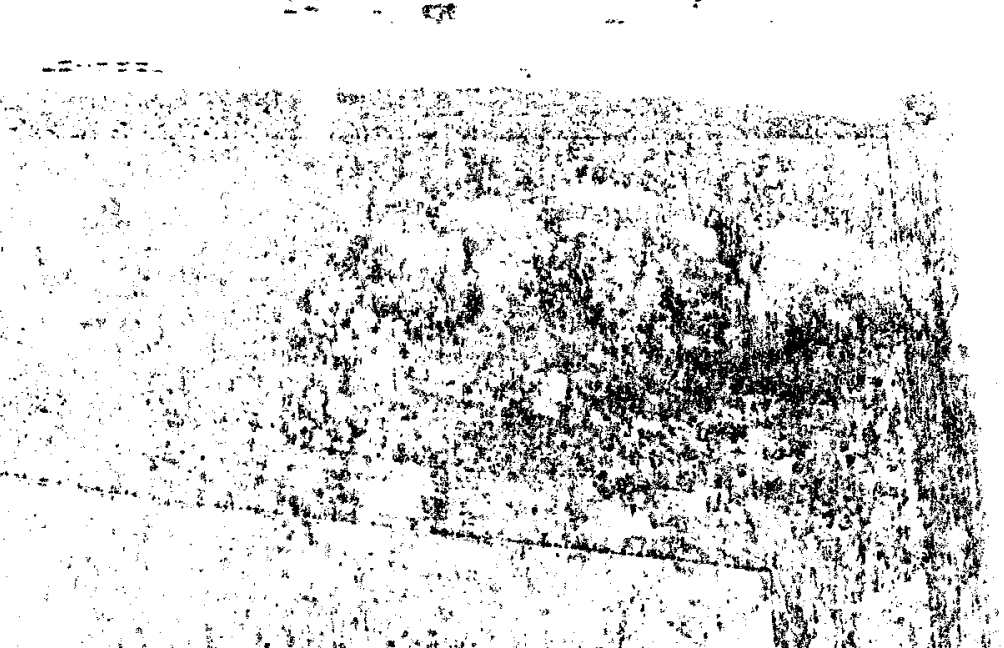

Why HALL

nto

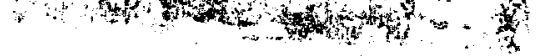

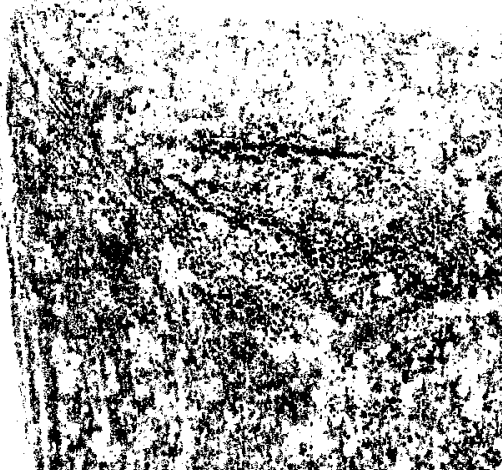




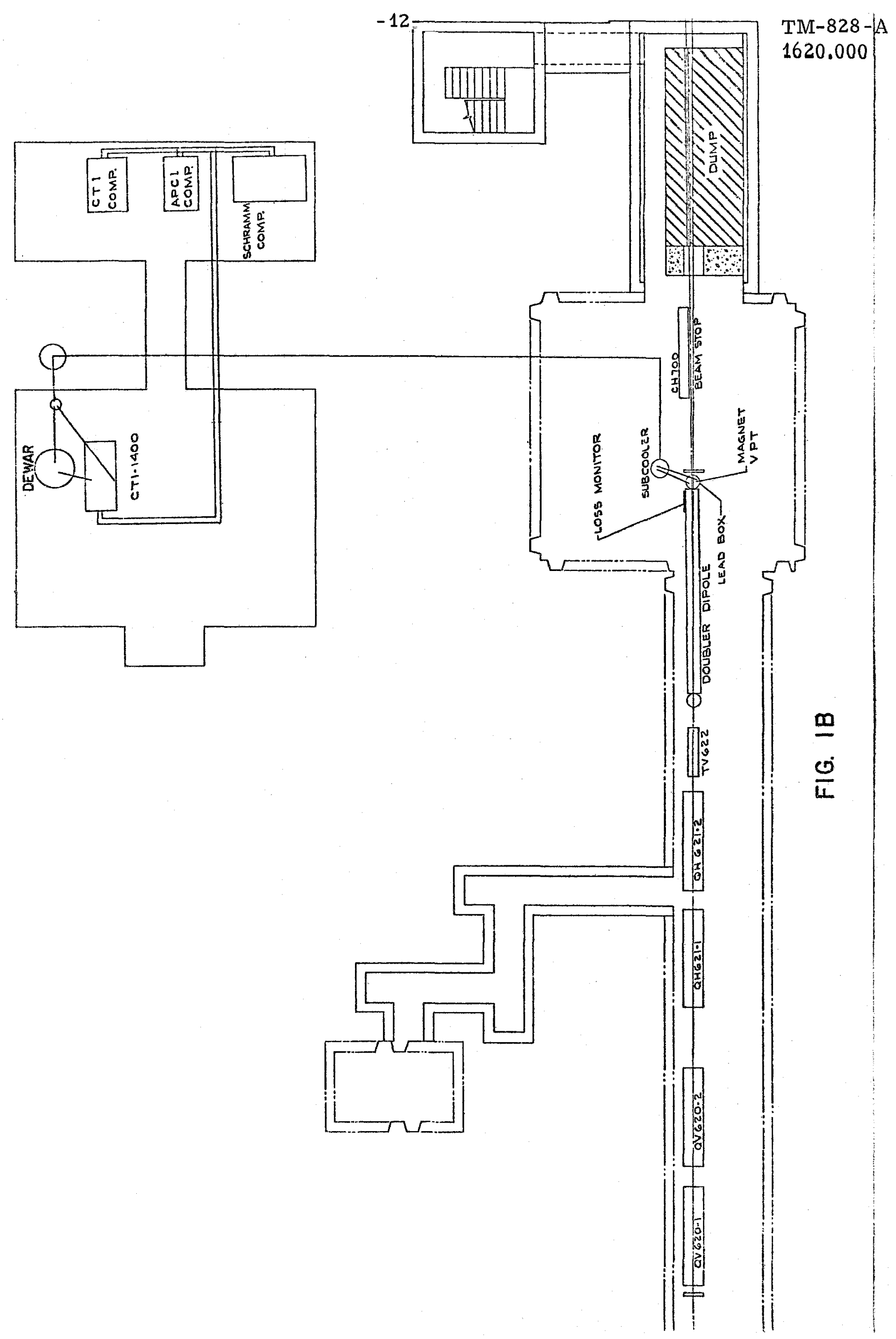




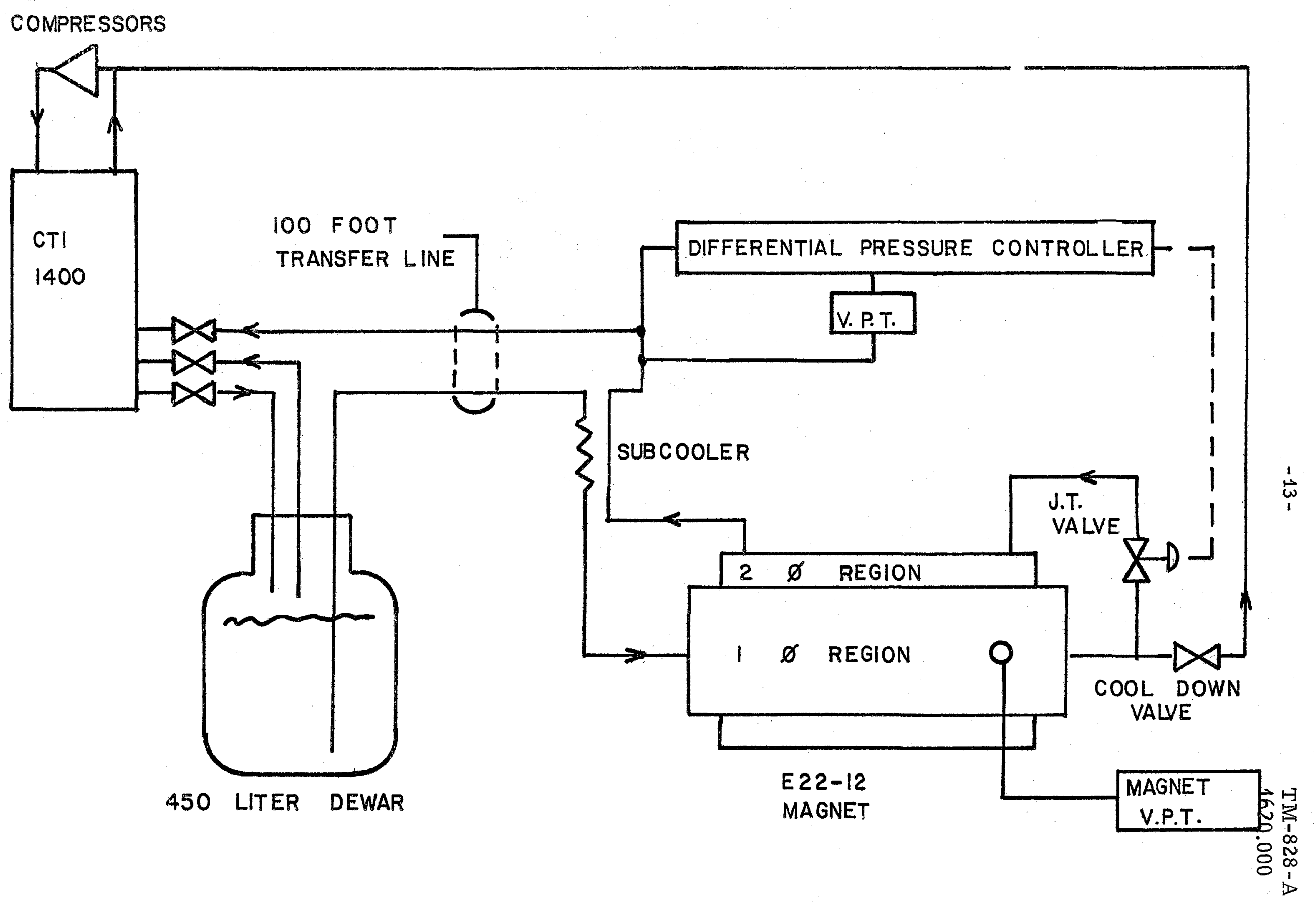

SCHEMATIC OF REFRIGERATION SYSTEM

FIGURE 2A 


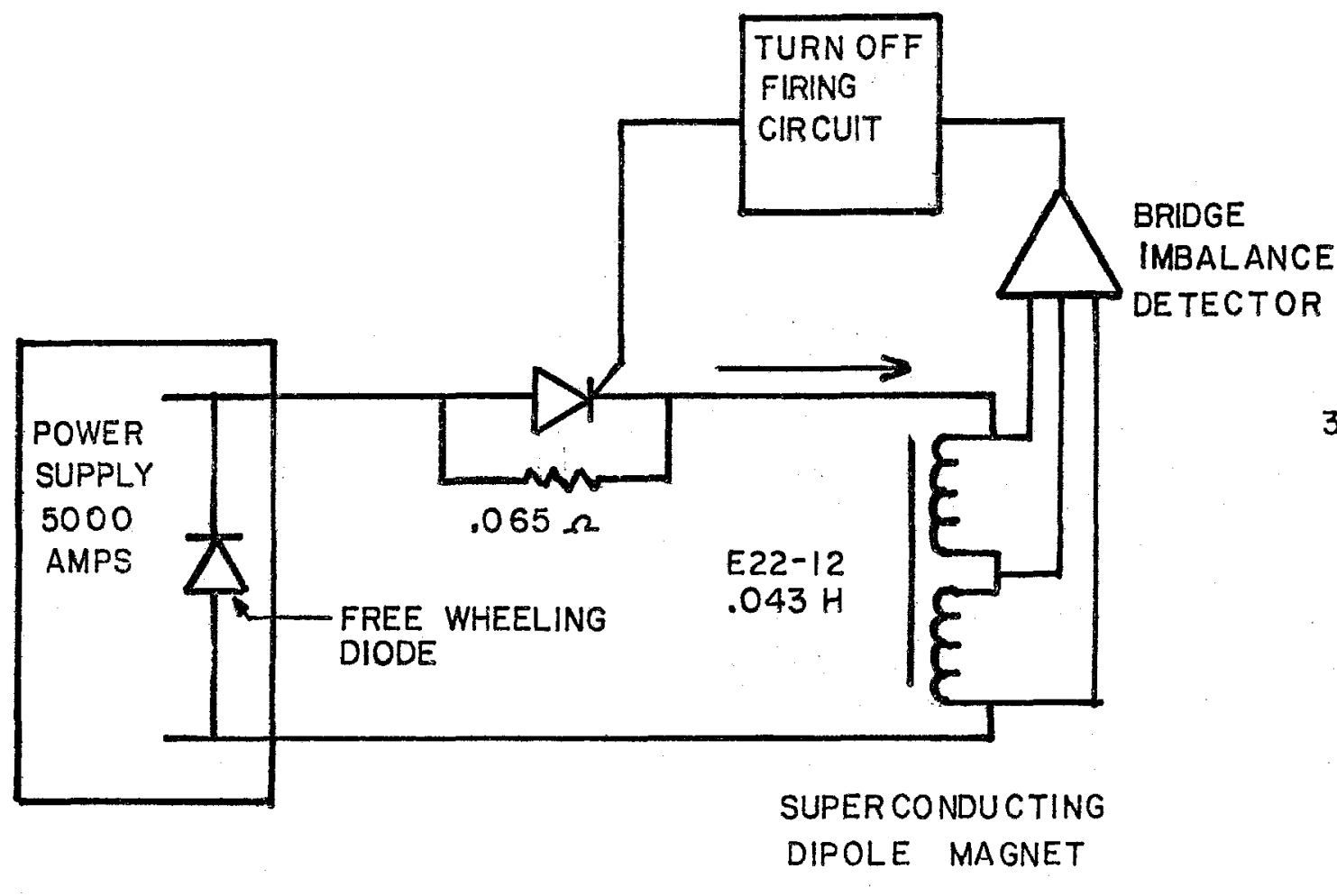

QUENCH DETECTOR AND PROTECTION CIRCUITRY

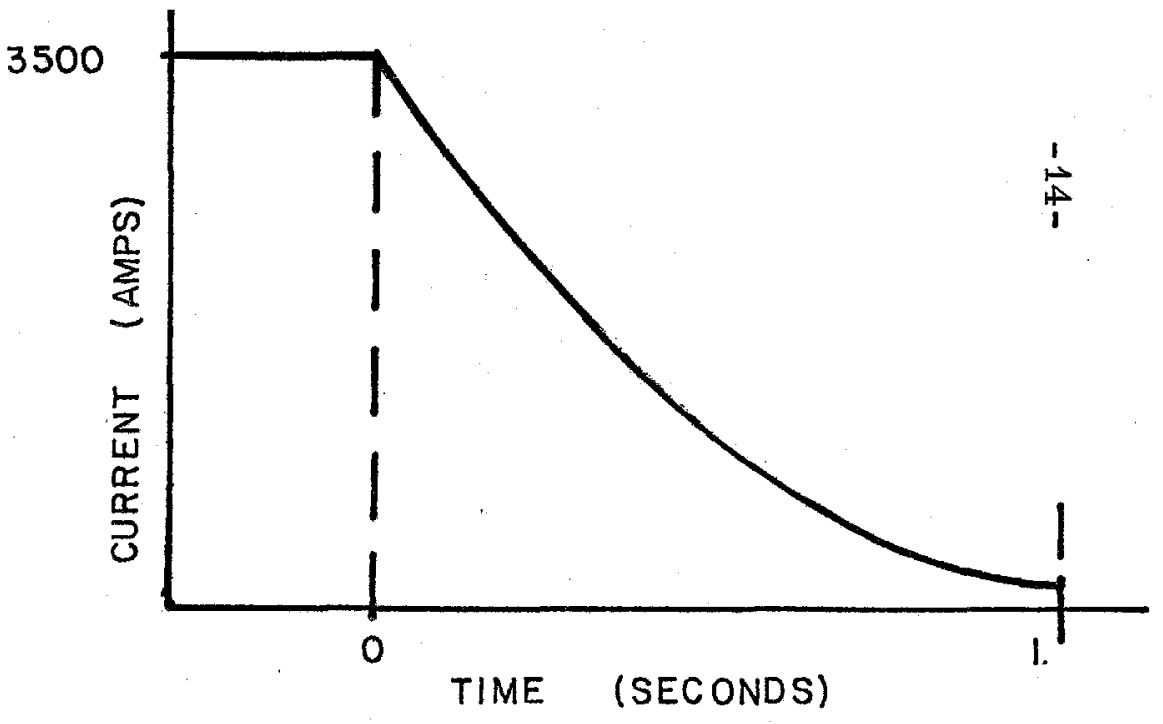

MAGNET CURRENT VERSUS

TIME DURING QUENCH

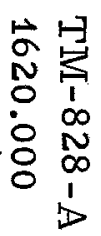




\section{GEOMETRY I}

UPSTREAM

END E22-12

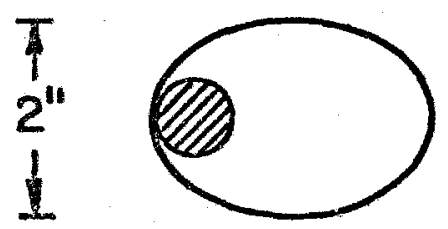

PROTON BEAM $18 \mathrm{MM} \times 15 \mathrm{MM}$

$$
1-2.9^{\prime \prime} \rightarrow
$$

INCIDENT $400 \mathrm{GEV} / \mathrm{C}$ PROTONS

SCHEMATIC

PLAN VIEW

ORIENTATION E22-12

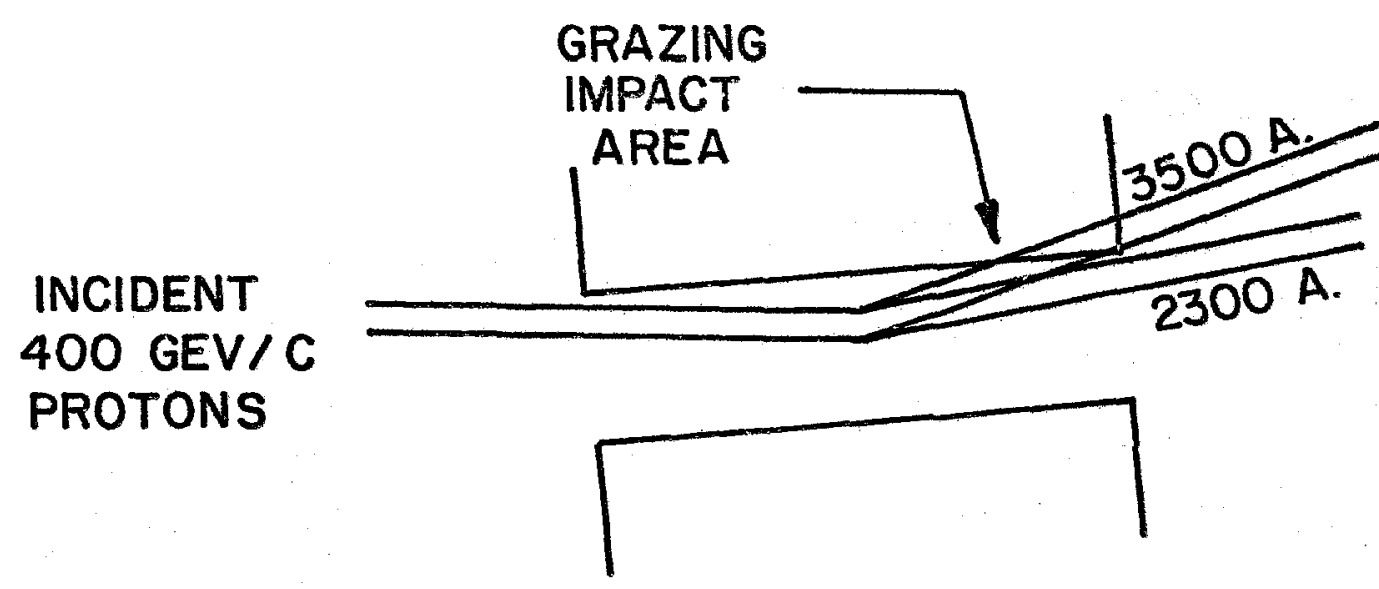

FIG. 3A

\section{GEOMETRY II}

UPSTREAM END

E22-12

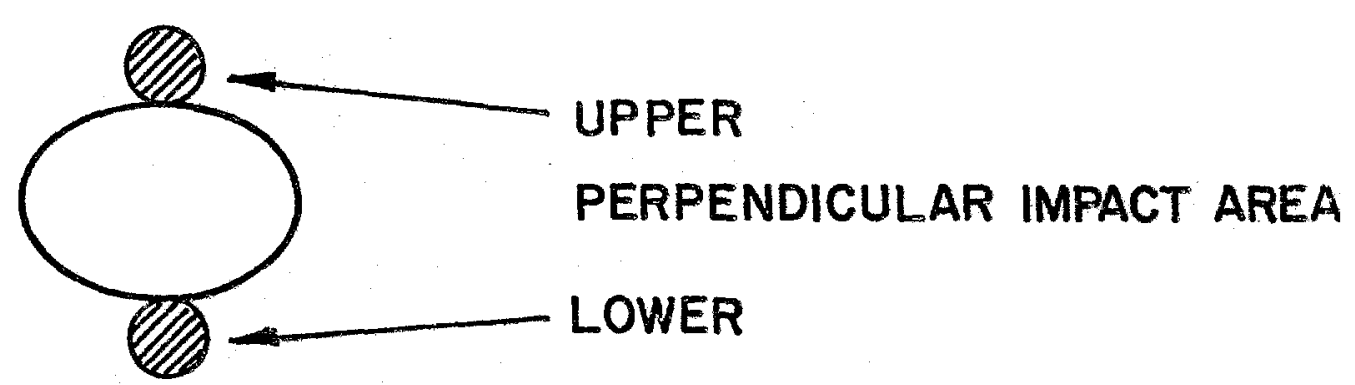

FIG 3B 
E22-12 MAGNET CROSS SECTIOI

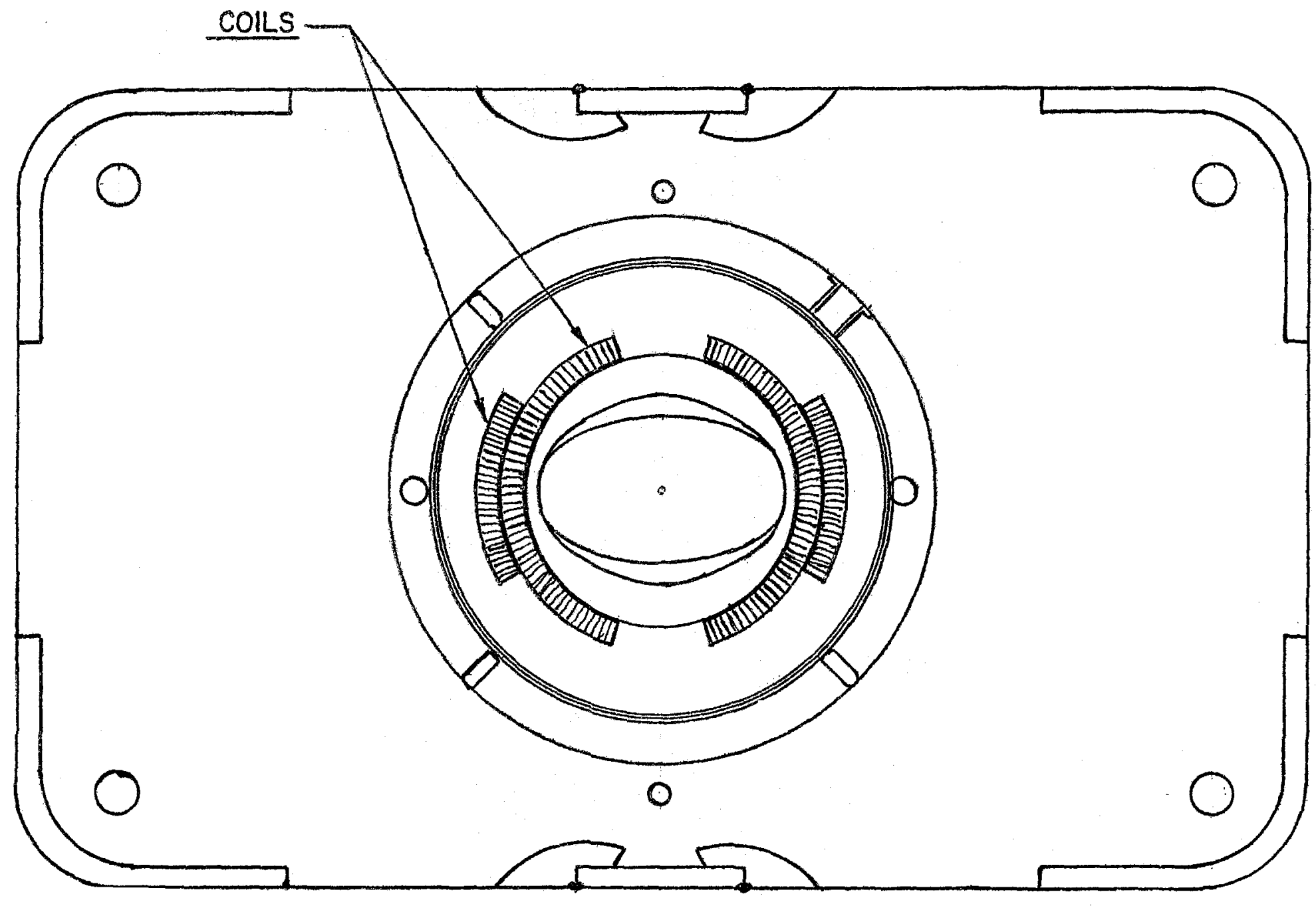




\section{GEOMETRY I \\ 3500 AMPS}

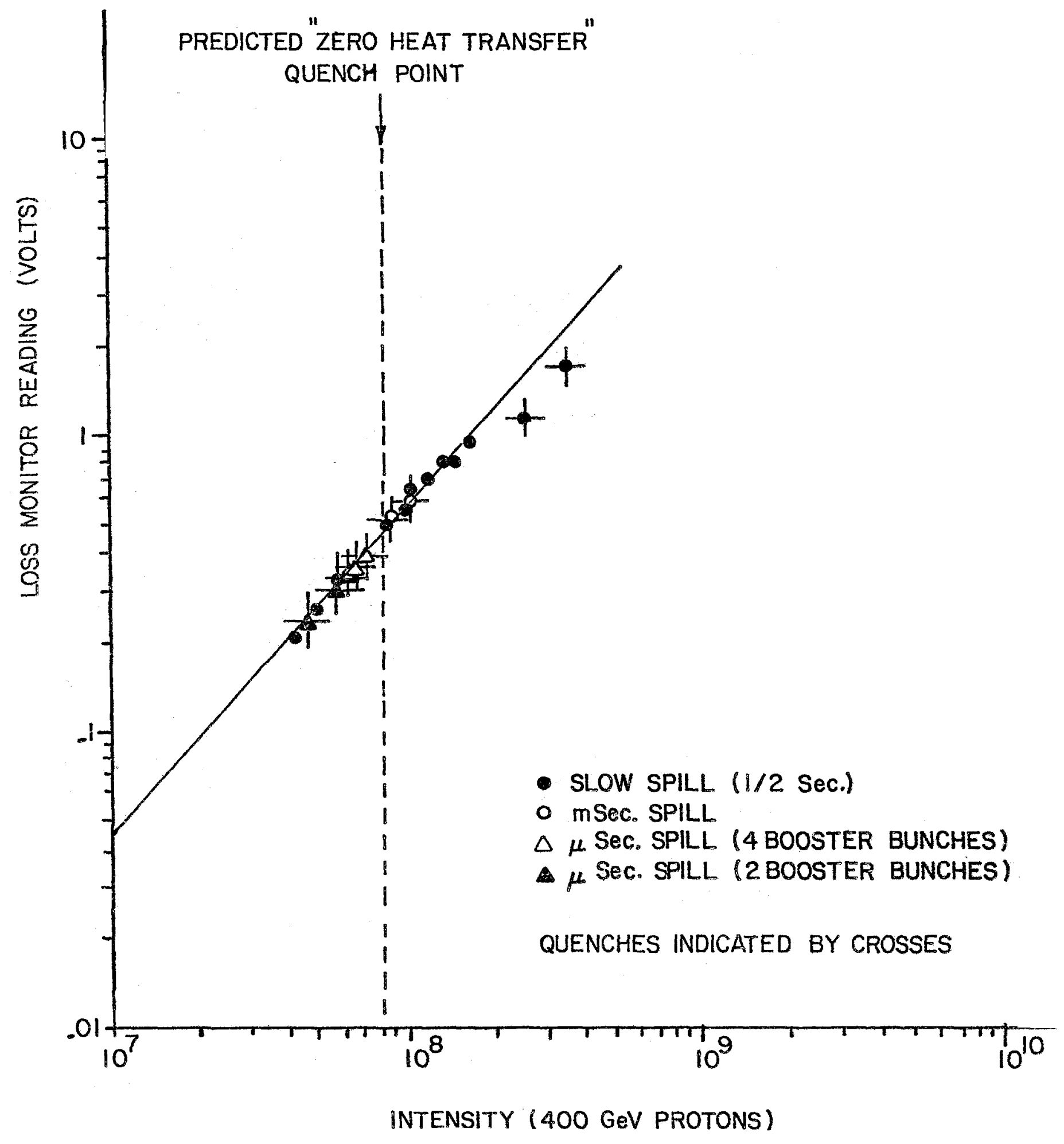

FIGURE 4 
GEOMETRY II

( 3500 AMPS)

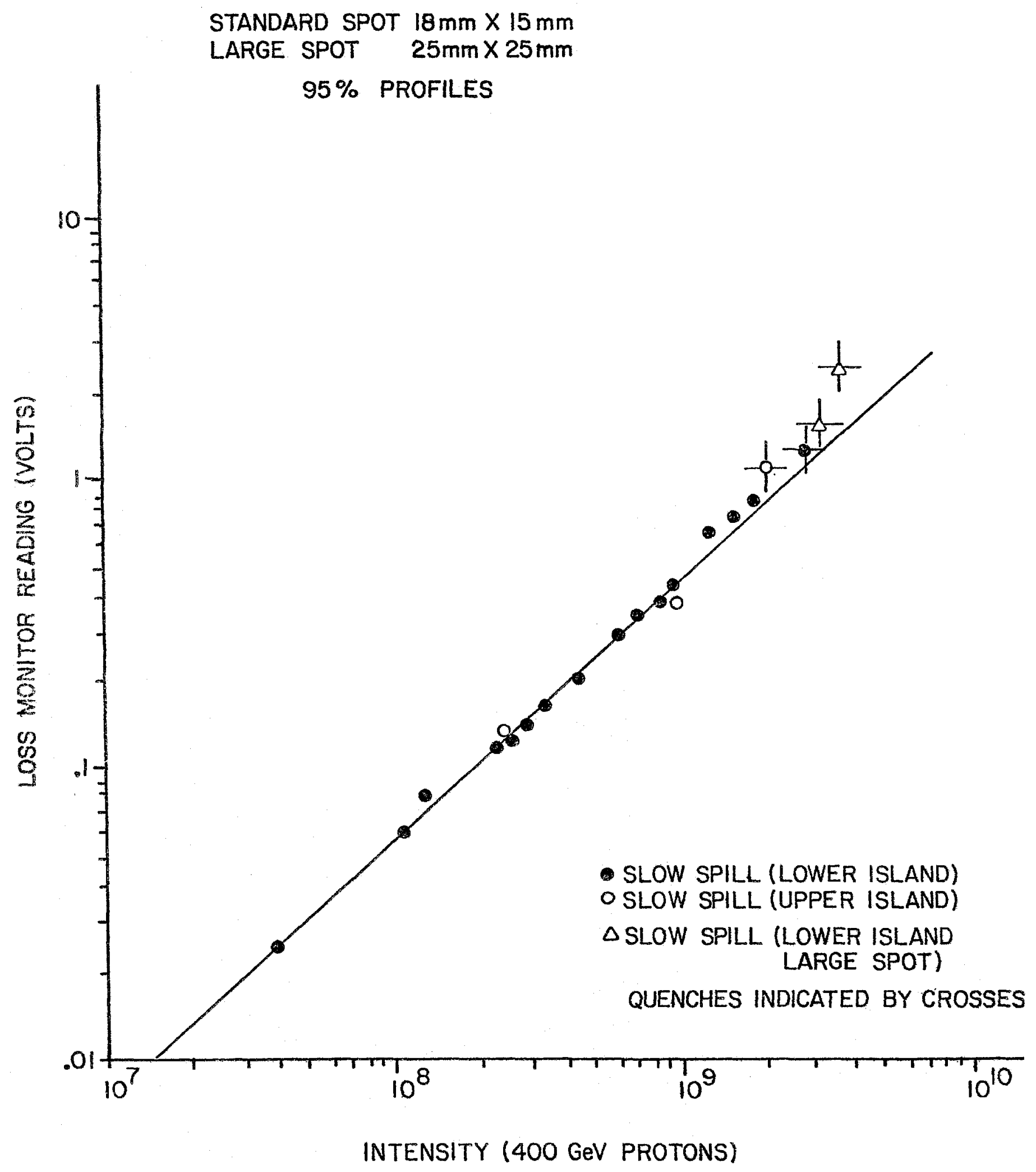

FIGURE 5 


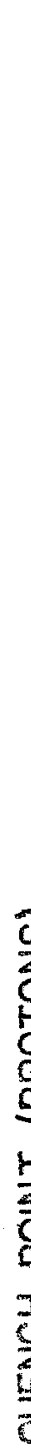

GEOMETRY II

DOUBLER DIPOLE QUENCH POINT

VS DIPOLE CURRENT

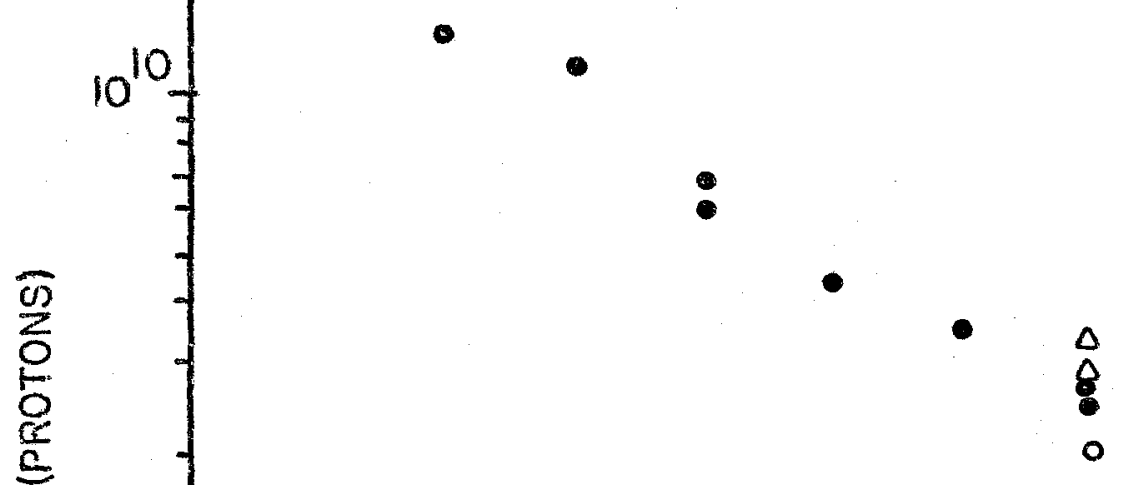

$0^{8}$

- SLOW SPILL (LOWER ISLAND)

- SLOW SPILL (UPPER ISLAND)

$\triangle$ SLOW SPILL (LOWER ISLAND.

LARGE SPOT)

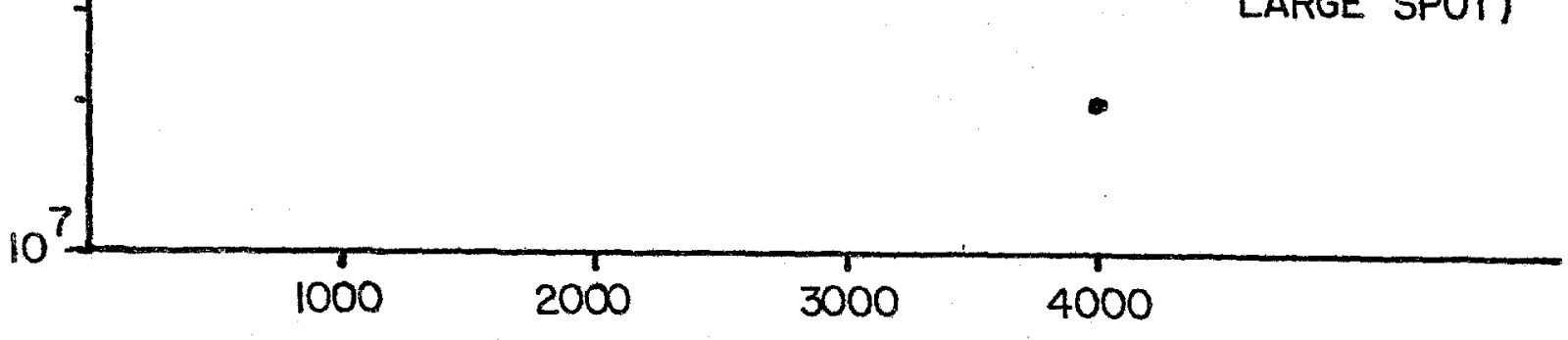

DIPOLE CURRENT (AMPS)

FIGURE 6 